\title{
Calcipotriol: A promising treatment for hand eczema
}

\author{
Ratchasin Pongprasert ${ }^{1 *}$, Premjit Juntongjin ${ }^{2}$ \\ ${ }^{1,2}$ Division of Dermatology, Chulabhorn International College of Medicine, Thammasat University, Pathumthani, Thailand
}

\author{
Key Words: \\ Hand eczema \\ Calcipotriol ointment \\ Desoximetasone ointment \\ Treatment
}

Received: 12 April 2017

Accepted: 20 May 2017

Published: 30 June 2017

\begin{abstract}
Hand eczema is a common dermatologic condition that has a poor response to conventional therapies. Calcipotriol, a topical vitamin $\mathrm{D}$ analog, has been approved to treat psoriasis and was reported to achieve a beneficial effect in the treatment of many other inflammatory diseases. This study aims to assess the efficacy and safety of calcium ointment in treating chronic hand eczema. Six patients were treated with calcipotriol twice daily for two weeks. HECSI and the patient's self-assessment evaluated the efficacy. Any adverse reaction was recorded during the experiment. The result revealed a statistical improvement of mean HECSI compared to the baseline after two weeks of treatment $(p=0.029)$. The only mild local side effect was observed. By subjects' evaluation, all the patients showed an improvement of clinical severity. The result can be implied that topical vitamin $D$ analog is safe and can be an alternative treatment for chronic hand eczema.
\end{abstract}

(C) 2017 The Author(s). Published by TAF Publishing.

\section{INTRODUCTION}

Chronic hand eczema refers to an eczematous process that exclusively or primarily involves hands which lasts for more than 3 months or relapses twice or more per year despite an adequate treatment and treatment adherence [1]. It is a common problem in the general population and has a substantial health, economic, and socio-medical impact on individuals. Two cross-sectional, web-based surveys performed in the United States informed that approximately $30 \%$ of patients with chronic hand eczema reported working impairment and productivity loss [2].

The pathogenesis of hand eczema is multifactorial etiologies since it has different causes and prognoses; thus, it makes the management complex. Many therapeutic modalities have been used for the treatment of chronic hand eczema, including skin protection program (patient education and avoidance of irritant or allergic factors), topical treatment (topical use of corticosteroids alone or in combination with keratolytic agents such as retinoic acid, emollients, calcineurin inhibitors) and even systemic treatment (systemic corticosteroid, methotrexate, azathioprine, etc.), and physical therapy (UVB, UVA, iontophoresis) [3].

Most of them show satisfactory result but prone to induce side effects [4, 5]. Topical vitamin $\mathrm{D}$ analogues are synthetically modified side-chain of $1 \alpha, 25$ - dihydroxyvitamin D3. They specifically bind to the Vitamin D Receptor (VDR) present on keratinocytes, melanocytes, and various inflammatory cells. Therefore, topical vitamin D analogues can modulate the epidermal proliferation, influence keratinocyte differentiation, and inhibit

\footnotetext{
${ }^{*}$ Corresponding author: Ratchasin Pongprasert

${ }^{\dagger}$ Email: ratchasinrp@gmail.com
} 
the inflammatory effect $[6,7]$. Calcipotriol, a topical vitamin D analogue, has been widely used in the treatment of psoriasis and was reported to achieve a beneficial effect in the treatment of other inflammatory diseases which share several pathogenic characters (impaired differentiation and increased proliferation of keratinocyte) $[8,9]$. The most common side effects after prolonged or excessive application are skin irritation and rarely hypercalcemia in patients with concomitant renal impairment $[10,11,12]$.

A systematic literature review conducted in 2014 of total 165 papers for the off-label uses of topical vitamin D analogues in the treatment of dermatologic disease other than psoriasis [9]. A moderate to strong recommendation was given for various types of ichthyoses, morphea, pityriasis alba, prurigo nodularis, and polymorphous light eruption when used as a monotherapy and for vitiligo when used in combination with corticosteroids and phototherapy $[8,9,10,11]$.

\section{MATERIALS AND METHODS}

\section{Subject}

This investigation was a prospective, pre-post treatment comparison. The study consisted of 6 patients aged more than 20 years old and diagnosed with Chronic Hand Eczema (CHE) (Duration $>3$ months or Recurrent $\geq 2$ episodes / year) of both the hands [13, 14].

The exclusion criteria included those who have other dermatologic condition of the hand or known allergy to calcipotriol ointment or, patients who have been using topical corticosteroid within the last 2 weeks or any systemic treatment within the last 4 weeks and pregnancy or lactation.

\section{Treatment Intervention}

Both hands of the patients were treated with calcipotriol ointment. The patients were advised to apply the ointment only at the lesion on each hand. Other emollients or any cream were not allowed on their hands during the period of study.

Pre-treatment photographs were taken. Patients underwent pretreatment self-evaluation and were assessed for the severity of their hands using HECSI by a dermatologist.

\section{Evaluation}

Clinical assessments were performed by the same investigator at the baseline before starting the experiment, then at the second week after the treatment. HECSI score was used to evaluate the clinical improvement at the baseline and at 2-week following the topical application.

Total HECSI score can range from 0 to 360 . It depends on the severity and the extension of the clinical symptoms at each location of the hands. For each location, the affected area was given a score from 0 to $4(0,0 \% ; 1,1-25 \% ; 2,26-50 \% ; 3,51-75 \%$, and $4,76-100 \%$ ) for the extent of clinical features, including erythema, infiltration or population, vesicles, fissures, scaling, and oedema. Finally, the score given for the extent for each location was multiplied by the total sum of the intensity of each clinical feature. The total sum is called the HECSI score.

Possible adverse effects related to the treatment including erythema, burning or stinging, and scaling were recorded. Patients were also asked to assess the improvement of their hands using Quartile grading score. The score ranges from 0 to 4. 
TABLE 1. Quartile grading score

\begin{tabular}{lc}
\hline \hline Grading score & Description (\% of improvement) \\
\hline 0 & 0 \\
1 & $1-25$ \\
2 & $26-50$ \\
3 & $51-75$ \\
4 & $>75$ \\
\hline \hline
\end{tabular}

\section{Statistical Analysis}

SPSS version 21.0 (SPSS Inc, Chicago, IL, USA) was used to conduct the statistical analysis. The statistical significance of mean total HECSI changed compared with the baseline within the treated sides was determined by using Wilcoxon matched pairs test. $p$-value at less than 0.05 was determined as statistical significance.

\section{RESULTS}

\section{Subjects}

Six patients completed this study. Two-third of the subjects were female $(n=4)$, and onethird were male $(n=2)$. The mean age of the subjects was 47 years (range 26-59 years old). Mean duration of hand eczema among the subjects was 8.62 month. Clinical variants of patients' hands were mostly hyperkeratotic palmar eczema and hyperkeratotic concomitant with chronic fissured hand eczema (66.7\%) followed by pulpitis type (33.3\%).

\section{Outcomes}

Clinical outcome was assessed by dermatologist by using HECSI. At the baseline, the mean HECSI score was 34+/-5.37. After treated with calcipotriol ointment on the affected areas for 2 weeks, the mean HECSI score was 21.6+/-5.88. It has been shown that there was a statistically significant improvement $(p=0.029)$ following the calcipotriol application.

TABLE 2. Demographic data of the subjects

\begin{tabular}{llccl}
\hline \hline Number & Gender & Age (year) & Duration of disease (month) & Clinical variants \\
\hline 1 & Female & 59 & 15 & Hyperkeratotic + Chronic fissured hand eczema \\
2 & Male & 54 & 7 & Hyperkeratotic palmar eczema \\
3 & Female & 26 & 7 & Pulpitis \\
4 & Male & 41 & 6 & Hyperkeratotic palmar eczema \\
5 & Female & 41 & 5 & Pulpitis \\
6 & Female & 61 & 10 & Hyperkeratotic + Chronic fissured hand eczema \\
\hline \hline
\end{tabular}

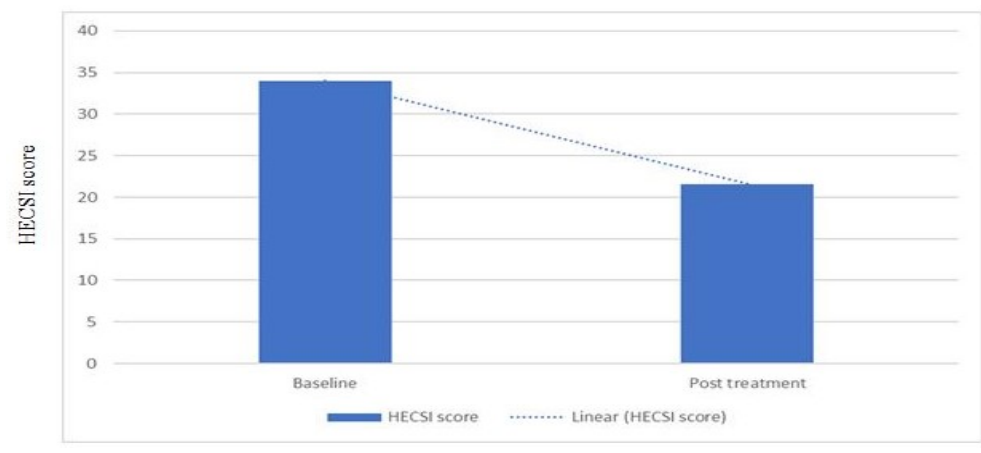

FIGURE 1. HECSI score at baseline and at 2-week post calcipotriol application 


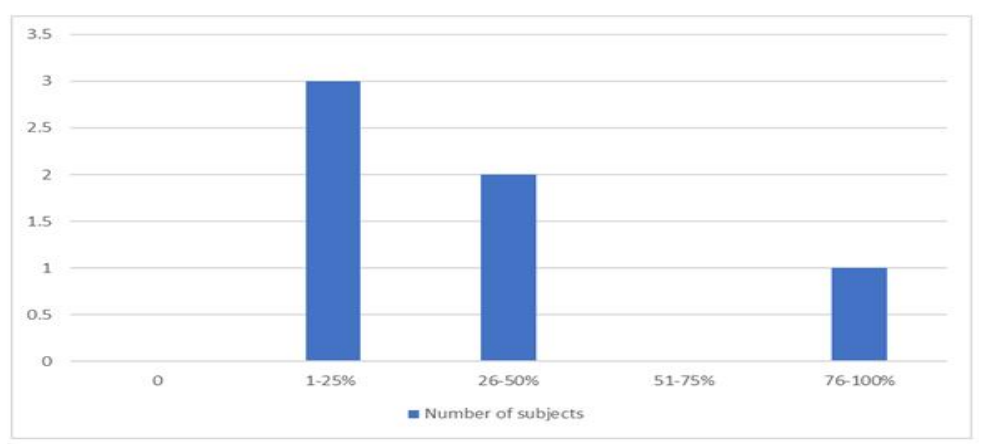

FIGURE 2. Number of subjects at different quartile grading scale for the improvement following the calcipotriol application

Patients were also evaluated for the improvement of their hands using quartile grading score. By patients' assessment, all patients recognized some improvement of both the hands. Interestingly, one patient reported more than $75 \%$ improvement after the application of calcipotriol ointment for 2 weeks. One-third of the subjects graded 26 to $50 \%$ improvement, and half of the participants experienced at least 1 - 25\% improvement.

\section{Safety}

There was no serious side effect or any systemic side effect in this study. Only few localized side effects were detected during the treatment. Scaling was the common side effect. However, most of them were only mild degree which exhibited in $83.33 \%$ of the patients. Only one patient accounting for $16.66 \%$ experienced moderate scaling. Scaling occurred one or two weeks after the treatment. Nevertheless, the scaling was spontaneously resolved.

\section{DISCUSSION}

Many treatment modalities have been used in the treatment of chronic hand eczema. However, due to its multifactorial etiologies and various morphologies, the management seems to be complex, and some of the them are prone to induce serious adverse effect after chronic application. Topical vitamin D derivative was approved in the treatment of adult plaque-type psoriasis and was reported to have a beneficial effect in the treatment of other inflammatory dermatoses which share pathogenic character of impaired keratinocyte diffentiation and proliferation $[8,9]$. Former studies demonstrated that twice daily application of topical vitamin $\mathrm{D}$ analogues has been reported to have an excellent therapeutic effect in the clinical cases reported of 6 patients with hyperkeratotic palmoplantar eczema [15].

The lesions almost disappeared after 2 to 8 weeks of treatment in 4 patients and highly improved after 7 weeks of treatment in patient [15]. This can be implied that topical vitamin $\mathrm{D}$ analogues may have the potential to be used as an alternative treatment for chronic hand eczema and, therefore, needs further studies to give stronger statistical evidence. Our study was a prospective pre-post treatment comparison study to evaluate the efficacy and safety of Calcipotriol ointment in the treatment of chronic hand eczema. We assessed the clinical severity of the lesion by using digital photographs and HECSI at the baseline and every follow-up. What makes our study different from the previous study of Egawa et al. [15] which demonstrates an excellent therapeutic response of hyperkeratotic hand eczema to topical vitamin $\mathrm{D}$ analogue is that the clinical severity in our study can be 
followed as scoring though we use HECSI as an objective asessement. Moreover, patients in our study could also evaluate the severity of their hands by using Quartile grading score in every visit which could determine the clinical significance.

The result of our study reveals an excellent therapeutic response of chronic hand eczema after treated with calcipotriol ointment for two weeks. The reduction of mean HECSI compared with the baseline in all the patients demonstrated a marked statistically significant reduction $(p<0.05)$ with very few side effects. The clinical severity showed a significant improvement ( $p=0.029$ ) even after only two weeks of treatment with around $34 \%$ reduction in mean HECSI compared with the baseline score. In addition, patients also evaluated the improvement of their hands by using Quartile grading score. This score helped the patients determine the severity of their hands in terms of how much they think their hands were improved.

All subjects could notice some improvement. This can imply that in patients' opinion, calcipotriol ointment can provide a beneficial improvement for their hands. Similar to the former study by Egawa et al. [15], the result of our study shows a beneficial effect in hyperkeratotic type of hand eczema which is the most clinical characteristic found in the experiment. From the result our research has provided, further studies with a larger number of participants and longer duration of follow-up are suggested in terms of yielding more precision and accuracy. Regarding the side effects, there were few localized side effects detected during the treatment and there was no serious side effect or any systemic side effect found in this study.

The most common adverse reaction from calcipotriol ointment was mild scaling which exhibited in $83 \%$ of patients. This reaction may be the result of modulated keratinocyte differentiation effect of calcipotriol ointment. Hence, the application of topical emollient was suggested to reduce and solve this adverse reaction.

\section{CONCLUSION}

In conclusion, topical calcipotriol seems to offer an effective and safe alternative form of treatment for chronic hand eczema, especially in hyperkeratotic type which is the most common variant found in the study.

The result of this study revealed a marked statistically significant improvement of the clinical severity ( $p=0.029$ ) even after only two weeks of treatment with around $34 \%$ reduction in mean HECSI compared with the baseline. Moreover, as regard to the patients' perspective, calcipotriol ointment also seems to yield a satisfactory therapeutic efficacy without any systemic or serious side effect. Scaling was the most frequent side effect found in the study and was spontaneously resolved by the end of study.

\section{REFERENCES}

1. Menné T, Johansen JD, Sommerlund M, Veien NK. Hand eczema guidelines based on the Danish guidelines for the diagnosis and treatment of hand eczema. Contact Dermatitis. 2011; 65(1): 3-12.

DOI: $10.1111 / \mathrm{j} .1600-0536.2011 .01915 . \mathrm{x}$

2. Dibenedetti D, Baranowski E, Zelt S, Reynolds M, Sherrill B. Assessing United States patient and dermatologist experiences with severe chronic hand eczema. The Journal of Clinical and Aesthetic Dermatology. 2015; 8(11): 19-25. 
3. Diepgen TL, Andersen KE, Chosidow O, Coenraads PJ, Elsner P, English J, Agner T. Guidelines for diagnosis prevention and treatment of hand eczema. Journal Der Deutschen Dermatologischen Gesellschaft. 2015; 13(1): 77-85.

DOI: $10.1111 /$ ddg.12510_suppl

4. English J, Aldridge R, Gawkrodger DJ, Kownacki S, Statham B, White JML, Williams J. Consensus statement on the management of chronic hand eczema. Clinical and Experimental Dermatology. 2009; 34(7): 761-769.

DOI: $10.1111 /$ j.1365-2230.2009.03649.x

5. Kraft JN, Lynde CW. Moisturizers: What they are and a practical approach to product selection. Skin Therapy Letter. 2005; 10(5): 1-8.

6. Bissonnette R, Worm M, Gerlach B, Guenther L, Cambazard F, Ruzicka T, Brown TC. Successful retreatment with alitretinoin in patients with relapsed chronic hand eczema. British Journal of Dermatology. 2010; 162(2): 420-426.

DOI: $10.1111 / \mathrm{j} .1365-2133.2009 .09572 . x$

7. Nijsten TE, Stern RS. The increased risk of skin cancer is persistent after discontinuation of psoralen+ ultraviolet A: A cohort study. Journal of Investigative Dermatology. 2003; 121(2): 252-258. DOI: 10.1046/j.1523-1747.2003.12350.x

8. Holm EA, Jemec GB. The therapeutic potential of calcipotriol in diseases other than psoriasis. International Journal of Dermatology. 2002; 41(1): 38-43. DOI: 10.1046/j.1365-4362.2002.01339.x

9. Wat H, Dytoc M. Off-label uses of topical vitamin D in dermatology: A systematic review. Journal of Cutaneous Medicine and Surgery. 2014; 18(2): 91-108. DOI:10.2310/7750.2013.13109

10. De Kerkhof PV. In vivo effects of vitamin D3 analogues. Journal of Dermatological Treatment. 1998; 9(sup3): S25-S29.

11. Bury Y, Ruf D, Carlberg C, Hansen CM, Kissmeyer AM, Binderup L. Molecular evaluation of vitamin D3 receptor agonists designed for topical treatment of skin diseases. Journal of Investigative Dermatology. 2001; 116(5): 785-792.

DOI: $0.1046 / \mathrm{j} .1523-1747.2001 .01332 . x$

12. Fogh K, Kragballe K. Vitamin D3 analogues. Clinics in Dermatology. 1997; 15(5): 705-713.

DOI: $10.1016 / \mathrm{S} 0738-081 \mathrm{X}(97) 00021-7$

13. Ruzicka T, Lynde CW, Jemec GBE, Diepgen T, Berth-Jones J, Coenraads PJ, Cambazard F. Efficacy and safety of oral alitretinoin (9-cis retinoic acid) in patients with severe chronic hand eczema refractory to topical corticosteroids: Results of a randomized, double-blind, placebo-controlled, multicentre trial. British Journal of Dermatology. 2008; 158(4): 808-817. DOI: 10.1111/j.1365-2133.2008.08487.x

14. Duman N, Uzunali E. Clinical assessment of the severity of chronic hand eczema: Correlations between six assessment methods. The European Research Journal. 2015; 1(2): 44-48. D0I: 10.18621/eurj.2015.1.2.44

15. Egawa K. Topical vitamin D3 derivatives in treating hyperkeratotic palmoplantar eczema: A report of five patients. The Journal of Dermatology. 2005; 32(5): 381-386. DOI: 10.1111/j.1346-8138.2005.tb00911.x

— This article does not have any appendix. - 